\title{
A Case with Posterior-fossa Epidermoid Cyst Showing Audiovestibular Symptoms Caused by Insufficiency of Anterior Inferior Cerebellar Artery -Usefulness of Free DICOM Image Viewing and Processing Software-
}

Kenji Takasaki, M. D. ${ }^{1)}$, Hidetaka Kumagami, M. D. ${ }^{1)}$

Akiko Baba, M. D. ${ }^{1)}$, Daisuke Fujiyama, M. D. ${ }^{1)}$, Haruo Takahashi, M. D. ${ }^{1)}$

1) Department of Otolaryngology - Head and Neck Surgery, Nagasaki University Graduate School of Biomedical Sciences

Address:

Department of Otolaryngology - Head and Neck Surgery,

Nagasaki University Graduate School of Biomedical Sciences

1-7-1, Sakamoto, Nagasaki 852-8501, Japan

Correspondence / Reprint requests:

Kenji Takasaki, M. D.

Department of Otolaryngology, Head and Neck Surgery,

Nagasaki University Graduate School of Biomedical Sciences

1-7-1, Sakamoto, Nagasaki 852-8501, Japan

Phone: +81-95-819-7349, Fax: +81-95-819-7352

E-mail:ktakasa@nagasaki-u.ac.jp 


\section{Abstract}

A 58-year-old Japanese man suddenly suffered from vertigo. On

physical examination, left-beating horizontal torsional spontaneous nystagmus was

observed, of which the direction did not change with gaze. Other neurotological

examinations revealed findings within normal limit except the left-side sensorineural

hearing loss of approximately $32 \mathrm{~dB}$ on an average. Diffusion weighted magnetic

resonance imaging revealed no infarction in the brain, but demonstrated an epidermoid

cyst in the left cerebello-pontine cistern region. Using free digital imaging and

communications in medicine (DICOM) image viewing and processing software, it was

found that the epidermoid cyst clearly compressed the left anterior inferior cerebellar

artery (AICA). Therefore, we speculated that insufficiency of the left AICA caused his

audiovestibular symptoms. This new technique used in the present study was

considered useful when the responsible site of vertigo is suspected in the

cerebello-pontine angle, where anatomical relationships between the nerves and the

vessels are complicated. 


\section{Keywords:}

AICA insufficiency, Cerebello-pontine angle, DICOM image, Epidermoid cyst, Vertigo, Viewing and processing software 


\section{Introduction}

Vertigo is a common presenting symptom in patients with transient

vertebro-basilar distribution ischemia (1). The anterior inferior cerebellar artery

(AICA) supplies the anterolateral regions of the inferior pons, inner ear, and middle

cerebellum including the flocculus (2). AICA infarction is known to be associated

with hearing loss (HL), vertigo, facial weakness, ataxia, anesthesia, and Horner's

syndrome (3). To date, using magnetic resonance imaging (MRI), some authors

reported AICA infarction showing only HL and vertigo as clinical signs without other

symptoms $(4,5)$; however, there has been no report that AICA insufficiency, especially

with the compression by a mass lesion, caused only HL or vertigo.

Recently, as digital imaging and communications in medicine

(DICOM) of computed tomography (CT) and MRI, and viewing and processing

software have advanced (6), it has become possible to observe and measure structures

seen in the CT and MRI three-dimensionally in a way that meets our needs $(7,8)$.

Using free DICOM image viewing and processing software, we reported a case whose

left-side AICA was compressed by an epidermoid cyst at the left cerebello-pontine 
cistern region and we speculated that the compression by the tumor caused AICA insufficiency, thus impairing the inner ear function and resulting in audiovestibular symptoms.

\section{Case Report}

A 58-year-old Japanese man with a long-standing history of hypertension presented with sudden onset of vertigo. He also complained of unsteady feeling. On physical examination, left-beating horizontal torsional spontaneous nystagmus was observed, of which the direction did not change with gaze. The eardrums were normal. He had not ophthalmoplegia, dysarthria, facial paralysis, facial numbness, Horner’s syndrome, nor cerebellar dysmetria.

Pure tone audiograms showed a sensorineural HL of approximately $32 \mathrm{~dB}$ on average in the left ear (Figure 1). Caloric tests using $2 \mathrm{ml}$ of ice water did not reveal a canal paralysis. Vestibular evoked myogenic potentials and eye tracking test were normal. Other neurotological and routine laboratory findings were all insignificant. 
T2-weighted, T1-weighted gadolinium-enhanced, and diffusion

weighted imaging (DWI) of MRI of the brain revealed no infarction. However, by three-dimensional constructive interference in steady-state (CISS) imaging, the mass was clearly demonstrated as low intensity in the left cerebello-pontine cistern region (Figure 2). The mass was not identified in conventional T1- and T2-weighted images because of the similarity in the signal intensity of the mass to that of cerebrospinal fluid (CSF), but was shown clearly by its increased signal intensity using DWI (Figure 3). From the absence of enhancement by gadolinium and similar signal intensity to the CSF, an epidermoid cyst was considered as the most possible diagnosis of the mass (9). Additionally, we used 0.5-mm-thick gapless MRI DICOM data, and an image viewing software (Virtual Place Liberty, Office Azemoto Ltd., Japan) on a personal computer to clarify the spatial anatomical relationships of this epidermoid cyst, and the vestibular nerve around the left internal auditory canal. The reconstructed CISS image demonstrated that the epidermoid cyst apparently compressed AICA at the left cerebello-pontine cistern region (Figure 4) and that it also had contact with the facial and trigeminal nerves. However, we could not recognize direct contact of the 
cyst either to the vestibular nerve or cochlear nerve in any image (Figure 5).

His vertigo disappeared over a week showing no paralytic nystagmus.

We followed the patient at every six months with neurotologic, and MRI examinations.

\section{Discussion}

In the process of the neurological diagnosis on a patient with vertigo,

we usually use selective diagnostic procedures including CT, MRI, DWI of MR, MR

angiography, and angiography (10). Acoustic schwannoma, meningioma, and

epidermoid cyst are the most common mass lesions around cerebellopontine angle

region, where an MRI usually provides more information than a CT. On T1-weighted

gadolinium-enhanced MRI, acoustic schwannoma demonstrates uniform enhancement

with $10 \%$ cystic necrosis. Meningioma also shows uniform enhancement with

susceptibility from calcified internal matrix and “dual tail sigh” on T2-weighted image.

Epidermoid cyst shows variable signal intensities. Typical MRI findings of

epidermoid cyst include similar or slightly increased signal intensity relative to that of

CSF on both T1- and T2-weighted images. DWI, which demonstrates differences in 
mobility of water, can differentiate solid components from fluid, therefore epidermoid cyst shows increased signal intensity (9).

Since the images obtained from those examinations are

two-dimensional, it is difficult to grasp three-dimensional relationship among the organs related with the pathogenesis of vertigo, especially the relationship between structures not running parallel each other, for example an artery, and a nerve. Recently, most CT and MRI are in the DICOM and viewing and processing software has been developed as a new imaging technique in the field of Radiology (6). With this technique, any arbitrarily reconstructed image desired can be obtained by changing the angle of the plane and by changing the target location. We previously reported new findings that we obtained regarding the spatial anatomy and dimensions of the eustachian tube and sphenoid sinus by using these techniques $(7,8)$. This is why we applied these techniques to clarify the pathogenesis of the vertigo of the present case by analyzing the spatial relationship among the tumor, nerves, and vessels.

In the present case, in spite of the left-beating horizontal torsional spontaneous nystagmus, and a sensorineural HL on the left ear, there was no 
neurotological or radiological findings indicating a brain infarction causing central vestibular dysfunction. We, therefore, suspected that the irritant nystagmus to the left was the left labyrinthine origin. Also, on CISS images, we found that the epidermoid cyst compressed the left-side AICA. The inner ear and the vestibulocochlear nerve are supplied exclusively from the labyrinthine artery, which stems from the AICA with few exceptions (2). Therefore, we speculated that the AICA inefficiency caused the irritant nystagmus originated from the left labyrinth (11).

AICA infarction is known to manifest audiovestibular symptoms caused by the inner-ear dysfunction as well as brainstem signs such as crossed sensory loss, lateral gaze palsy, facial palsy, or Horner's syndrome (3). There were a few reports of AICA infarction presenting only auditovestibular symptoms without other central signs $(4,5)$, but there has been no report that AICA insufficiency caused vertigo and HL of the inner ear origin. We might have diagnosed that the audiovestibular symptoms in the present case were caused by brain stem compression, or neurovascular compression of the eighth cranial nerves by the tumor, if we had not used new DICOM image-viewing and processing software. Therefore, these new useful techniques 
should be utilized fully when investigating the pathogenesis, and pathophysiology in cases with vertigo of unknown cause. 


\section{References}

1. Williams D, Wilson TG. The diagnosis of the major and minor syndromes of basilar insufficiency. Brain. 1962;85:741-74.

2. Kim HN, Kim YH, Park IY, Kim GR, Chung IH. Variability of the surgical anatomy of the neurovascular complex of the cerebellopontine angle. Ann Otol Rhinol Laryngol. 1990;99:288-96.

3. Adams RD. Occlusion of the anterior inferior cerebellarartery. Arch Neurol Psychiatry. 1943;49:765-70.

4. Son EJ, Bang JH, Kang JG. Anterior inferior cerebellar artery infarction presenting with sudden hearing loss and vertigo. Laryngoscope. 2007;117:556-8.

5. Lee H, Ahn BH, Baloh RW. Sudden deafness with vertigo as a sole manifestation of anterior inferior cerebellar artery infarction. J Neurol Sci. 2004;222:105-7.

6. Escott EJ, Rubinstein D. Free DICOM image viewing and processing software for your desktop computer: what's available and what it can do for you. Radiographics. 2003;23:1341-57.

7. Takasaki K, Takahashi H, Miyamoto I, Yoshida H, Yamamoto-Fukuda T, Enatsu K, 
Kumagami H. Measurement of angle and length of the eustachian tube on

computed tomography using the multiplanar reconstruction technique.

Laryngoscope. 2007;117:1251-4.

8. $\quad$ Enatsu K, Takasaki K, Jinnouchi S, Kase K, Kumagami H, Nakamura T, Takahashi

H. Surgical anatomy of the sphenoid sinus on the CT using the multiplanar reconstruction technique. Otolaryngol Head Neck Surg. in press.

9. Murakami N, Matsushima T, Kuba H, Ikezaki K, Morioka T, Mihara F, Inamura T, Fukui M. Combining steady-state constructive interference and diffusion-weighted magnetic resonance imaging in the surgical treatment of epidermoid tumors. Neurosurg Rev. 1999;22:159-62.

10. Bruzzone MG, Grisoli M, De Simone T, Regna-Gladin C. Neuroradiological features of vertigo. Neurol Sci. 2004;25 Suppl 1:S20-3.

11. Oas JG, Baloh RW. Vertigo and the anterior inferior cerebellar artery syndrome. Neurology. 1992;42:2274-9. 


\section{Legend}

Figure 1. Initial pure-tone audiogram showed slight sensorineural hearing loss on the left ear.

Figure 2. On the three-dimensional constructive interference in steady-state (CISS) magnetic resonance imaging, the mass $\left({ }^{*}\right)$ was clearly demonstrated as low intensity in the left cerebello-pontine cistern lesion. Arrowheads indicate internal auditory canals.

Figure 3. Diffusion-weighted axial magnetic resonance imaging showed high intensity in the left cerebello-pontine cistern region (*).

Figure 4. Using free DICOM image viewing and processing software, reconstructed CISS images of the left-side anterior inferior cerebellar artery (AICA) in the present case are demonstrated. "Plane A" is close to the coronal plane perpendicular to "Plane B” and "Plane C". "Plane B" is close to the sagittal plane perpendicular to "Plane A" and "Plane C". "Plane C" is close to the axial plane at the level of AICA, including the whole course of the presence of AICA loop. Arrows indicate the course of AICA loop, and asterisks indicate an epidermoid cyst compressing the AICA.

Figure 5. The cochlear and vestibular nerves demonstrated from the brainstem to the 
cochlea and vestibule, AICA and cochlea are shown in the reconstructed CISS image of the left-side internal auditory canal. The epidermoid cyst is not identified in this image. 


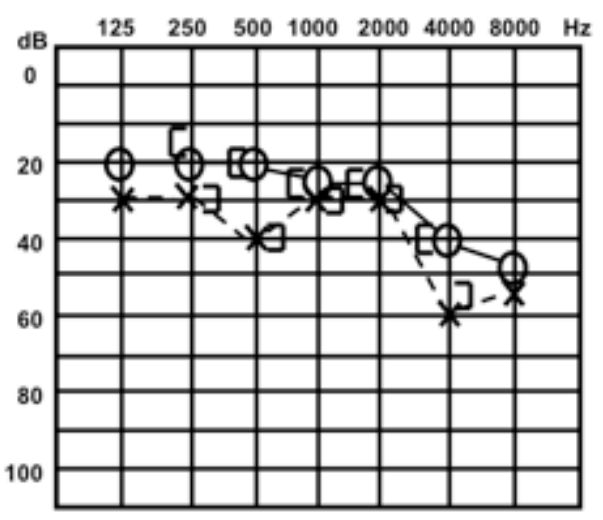

Figure 1

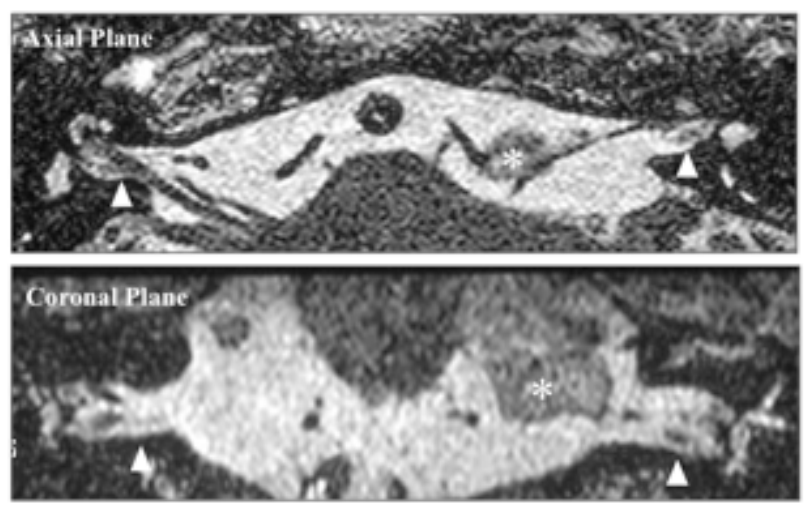

Figure 2 


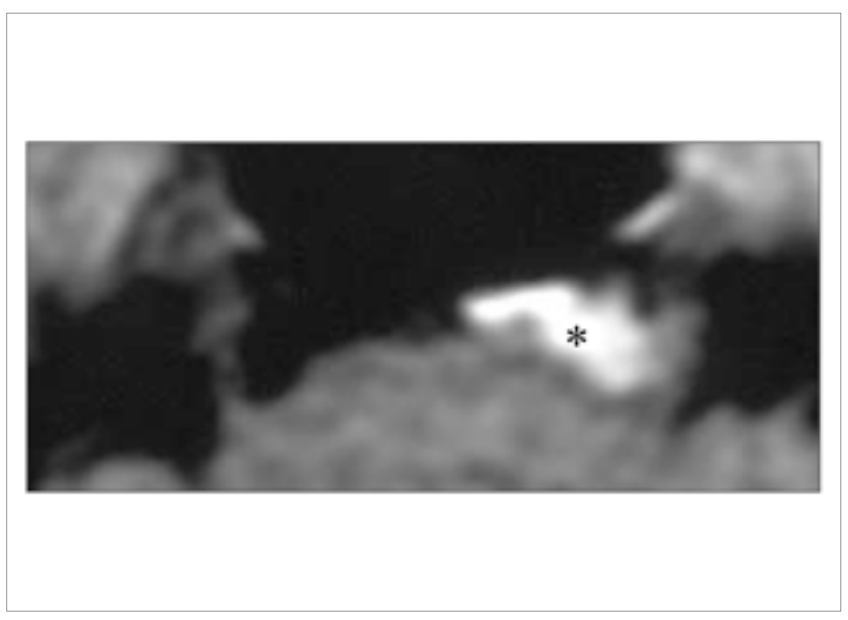

Figure 3

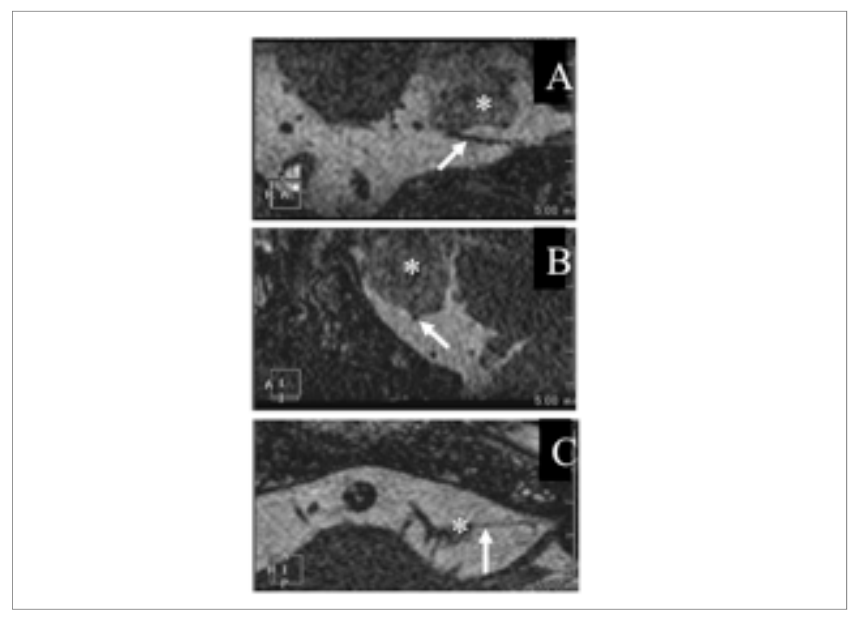

Figure 4 


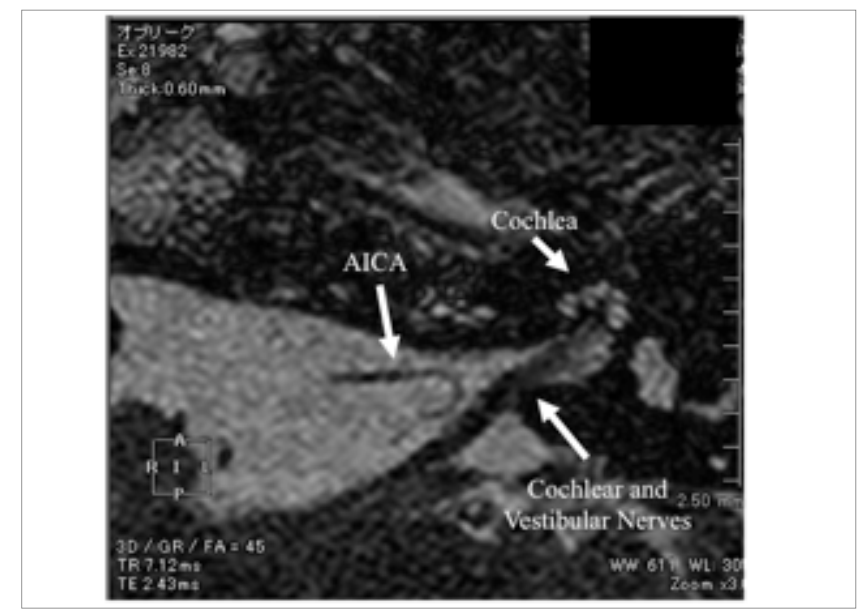

Figure 5 